\title{
Hofstadter Butterfly and Many body effects in epitaxial graphene superlattice
}

Wei Yang ${ }^{1,2}$, Xiaobo $\mathrm{Lu}^{1}$, Guorui Chen ${ }^{3}$, Shuang Wu${ }^{1}$, Guibai Xie ${ }^{1}$, Meng Cheng ${ }^{1}$, Duoming Wang $^{1}$, Rong Yang ${ }^{1}$, Dongxia Shi ${ }^{1}$, Kenji Watanabe ${ }^{4}$, Takashi Taniguchi ${ }^{4}$, Christophe Voisin ${ }^{2}$, Bernard Plaçais ${ }^{2}$, Yuanbo Zhang ${ }^{3}$, and Guangyu Zhang ${ }^{1 *}$

${ }^{1}$ Beijing National Laboratory for Condensed Matter Physics and Institute of Physics, Chinese Academy of Sciences, Beijing 100190, China.

${ }^{2}$ Laboratoire Pierre Aigrain, ENS-CNRS UMR 8551, Universités P. et M. Curie andParis-Diderot, 24 rue Lhomond, 75231 Paris Cedex 05, France.

${ }^{3}$ State Key Laboratory of Surface Physics and Department of Physics, Fudan University,Shanghai 200433, China.

${ }^{4}$ Advanced Materials Laboratory, National Institute for Materials Science, 1-1 Namiki, Tsukuba, 305-0044, Japan.

* Corresponding author: gyzhang@aphy.iphy.ac.cn

\section{Thermal stability of QH states}

Figure S1 shows thermal dependences of $\mathrm{QHE}^{1,2}$ at a fixed magnetic field $\mathrm{B}=9 \mathrm{~T}$. We transform the $\mathrm{x}$ axis $V_{g}$ into filling fraction $v$ by $v=\mathrm{n} / \mathrm{n}$, where $\mathrm{n}=\left(\mathrm{C}^{*} \Delta V_{g}\right)^{0.5} / \mathrm{e}$ is the carrier density and $\mathrm{n}$ ' $=B e / h$ is the number of quantum flux per unit area at a given magnetic field $\mathrm{B}$, while $\mathrm{C}$ is capacitance per unit area and e is the elementary charge. By doing so it is easy to identify two-fold lifted $\mathrm{QH}$ states $v=2 \mathrm{~N}$ with $\mathrm{N}=1,2,3 \ldots$ the landau level index from the $\mathrm{x}$ axis, indicated by resistance minima in Fig. S1a, while it is a little bit more complicated at the hole side $(\mathrm{N}=-1,-2$, $-3 \ldots)$ due to the strong influence of SDP. Note that we can identify $v=0$ state by transforming the resistance into conductance $\sigma_{x x}=R_{x x} /\left(R_{x x}{ }^{2}+R_{x y}{ }^{2}\right)$, with Hall resistance into account. Correspondingly, the $\mathrm{QH}$ gaps are instead indicated by conductance minima in Fig. S1b, and it is clear that there is a minimum at around $v=0$, suggesting $v=0 \mathrm{QH}$ state developed ${ }^{3}$ there, and it 
disappears once $\mathrm{T}>20 \mathrm{~K}$ as shown in Fig. S1c. We also included Hall conductance $\sigma_{x y}=$ $R_{x y} /\left(R_{x x}{ }^{2}+R_{x y}{ }^{2}\right)$ in Fig. S1d, and what is odd is that the $\sigma_{x y}$ around $\mathrm{v}=0$ is equal to $\sim 0.4 \mathrm{e}^{2} / \mathrm{h}$ instead of 0 , which may suggest an onset of $1 / 3$ fractional quantum Hall state.
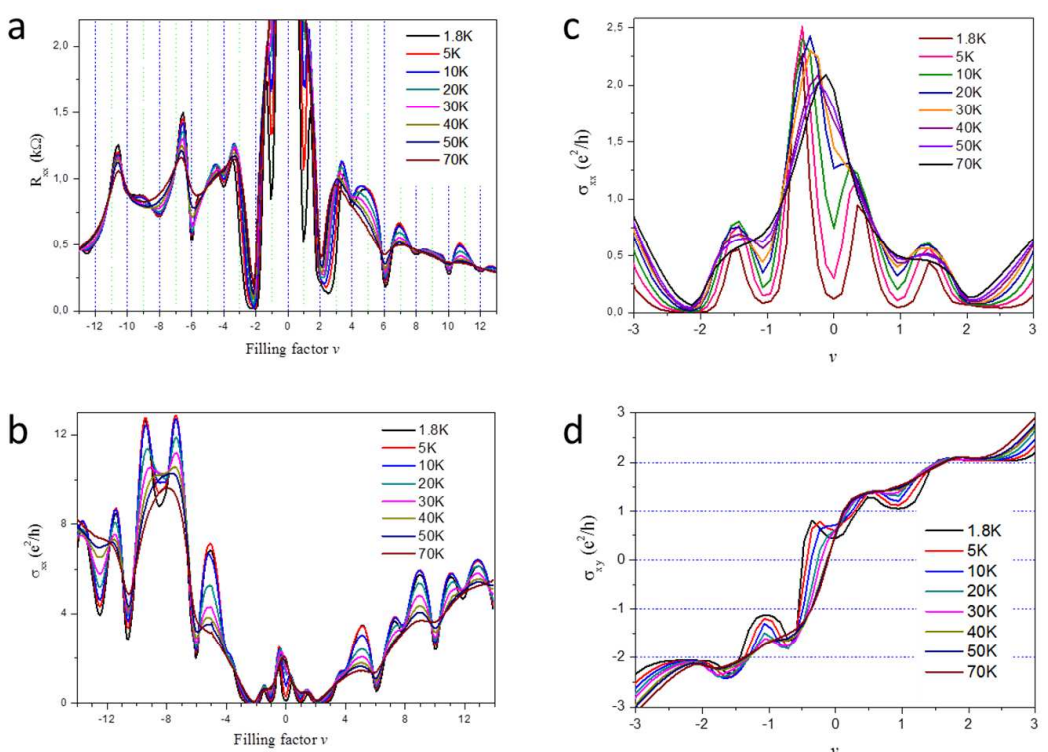

Figure S1. a-b are thermal dependence of longitudinal resistance $R_{x x}(\mathbf{a})$ and longitudinal conductance $\sigma_{x x}(\mathbf{b})$, with the $\mathrm{x}$ axis converted to filling factor for convenience in identifying quantized gaps. $\mathrm{c}$ is the zoomed-in plot of $\mathrm{b}$ around $\mathrm{DP}$, and $\mathrm{d}$ is the corresponding hall conductance.

A clearer and more comprehensive way to investigate the thermal stability of the QH states, including QH gaps from SDP and Hofstadter butterfly fractal states ${ }^{4}$, which are shown in Figure S2. It is clear that the LLs from DP and the LLs from SDPs, as well as the fractal Hofstadter butterfly states are well preserved at $T=20 \mathrm{~K}$ as shown in Fig. S1a and S1d, compared to Fig. $2 \mathrm{~b}$ at $T=1.8 \mathrm{~K}$ in the main text. However, when the temperature is increased further, symmetry broken states like $v=0, \pm 1,4 \mathrm{n}$ from DP, as well as $v_{s}=0, \pm 6, \pm 10$ from hSDP (superlattice Dirac point at hole side) and the fractal Hofstadter gaps ${ }^{4-8}$ at $\Phi / \Phi_{0}=1 / 3$ are greatly suppressed and they become barely visible at $T>30 \mathrm{~K}$. 

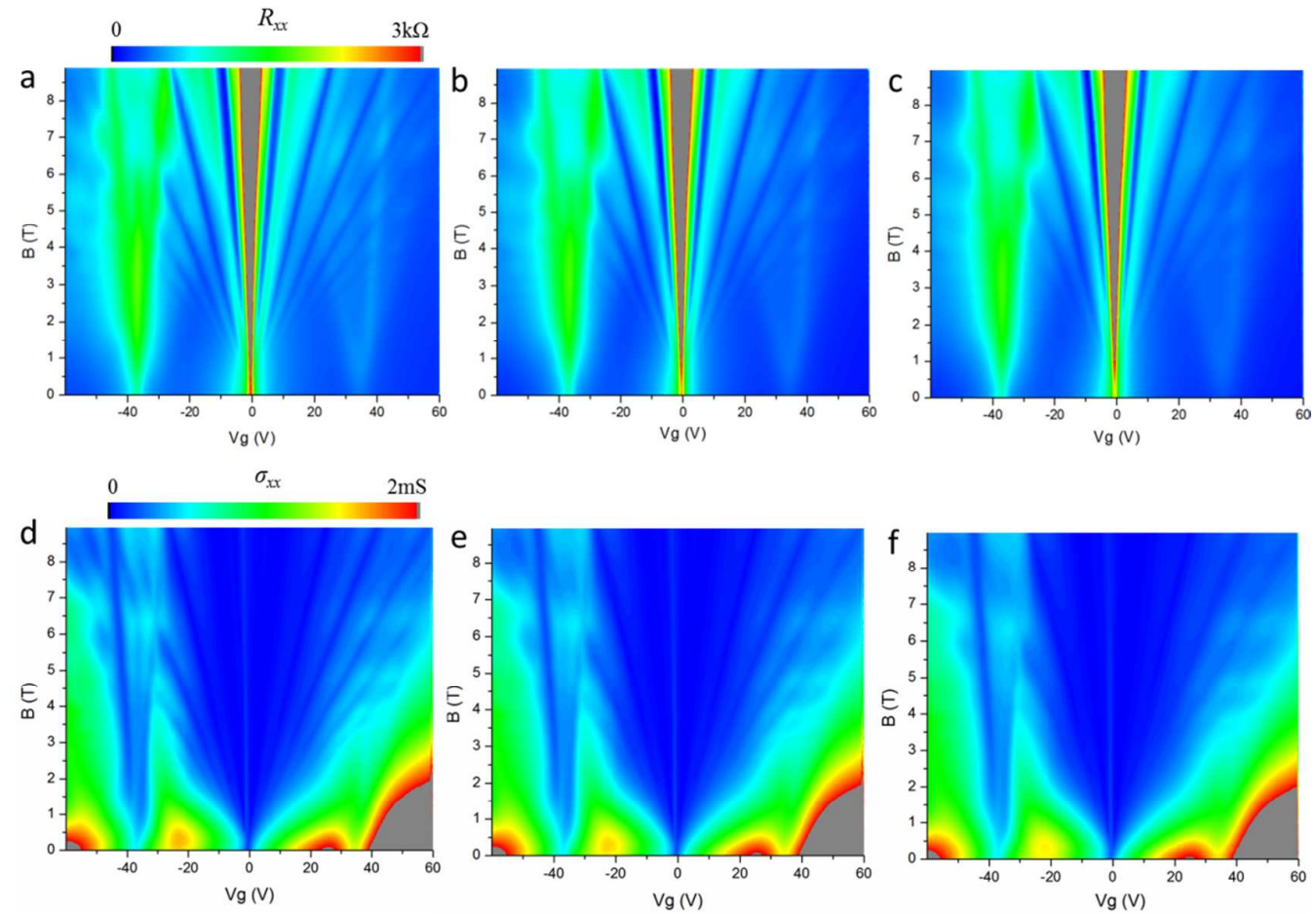

Figure S2. a-c are colored mapping of $R_{x x}$ for a temperature of $T=20 \mathrm{~K}, 30 \mathrm{~K}$, and $40 \mathrm{~K}$ respectively. d-f are the corresponding colored mapping of $\sigma_{x x}$.

\section{Onset of $v=0,1$ at low magnetic field}

Figure S3a is the zoomed-in image of Fig. 3a in the main text. The carrier density is chosen close to DP from $-1.5 * 10^{12} / \mathrm{cm}^{2}$ to $1.5 * 10^{12} / \mathrm{cm}^{2}$, and the color of $\sigma_{x x}$ mapping is chosen in log scale to identify LLs at small filling fraction. We can see that symmetry broken QH states $v=0,1$ are developed at a low magnetic field. To be specific, a line cut of $\sigma_{x x}$ mapping at around $3 \mathrm{~T}$ is shown in Fig. S3b, and the emergence of conductance minima at $v=0,1$ is a good indication of an onset of the symmetry broken states.
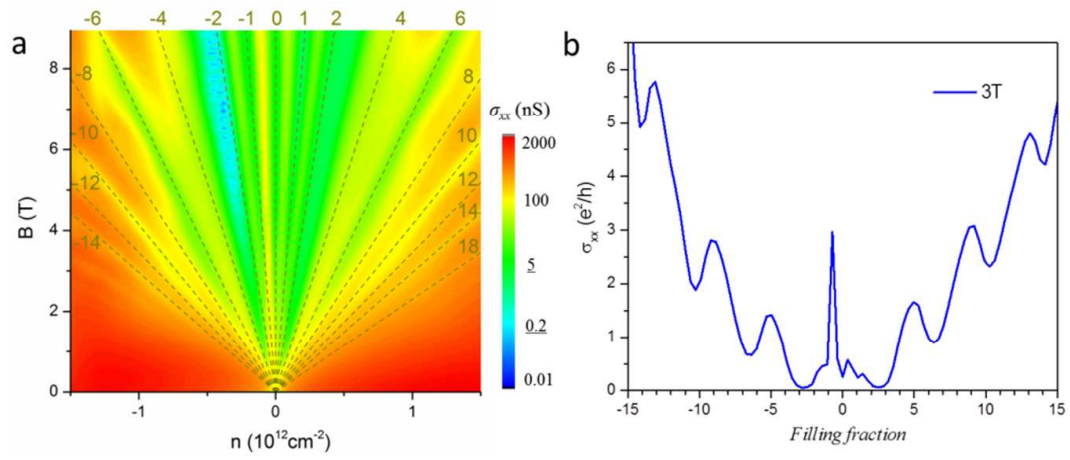

Figure S3. a is the zoomed in image of Fig.3a in the main text, and the dashed lines indicate gaped states at small filling fractions. $\mathbf{b}$ is a line cut of $\mathbf{a}$ at $B=3 \mathrm{~T}$. 


\section{Conductance at around $\Phi=\Phi_{1 / 3}$}

Figure S4 shows longitudinal conductance $\sigma_{x x}$ and Hall conductance $\sigma_{x y}$ in the vicinity of $\Phi=\Phi_{l / 3}$.

Fig. S4a-c are corresponded to conductance at hole side with $B=7.2 \mathrm{~T}, B=B_{1 / 3}=6.4 \mathrm{~T}$, and $B=5.6 \mathrm{~T}$ respectively, while Fig. S4d-f are the conductance at electron side. The change of Hall conductance from low carrier density to high carrier density is distinctively different from normal one, and it show peaks and valleys instead of plateaus. For example at $\mathrm{B}=\mathrm{B}_{1 / 3}=6.4 \mathrm{~T}$, we could see that there is a $\sigma_{x x}$ minimum with carrier density corresponding to a filling factor of -10 (as indicated by $\mathrm{x}$ axis in Fig. $4 \mathrm{~b}$ ), while the corresponding $\sigma_{x y}$ is around $-2 \mathrm{e}^{2} / \mathrm{h}$ instead of $-10 \mathrm{e}^{2} / \mathrm{h}$. Compared to the strong reconstruction of Hall conductance at hole side, $\sigma_{x y}$ at electron side in Fig. S4d-f only shows a small deviation from normal one thanks to the strong e-h asymmetry.

a

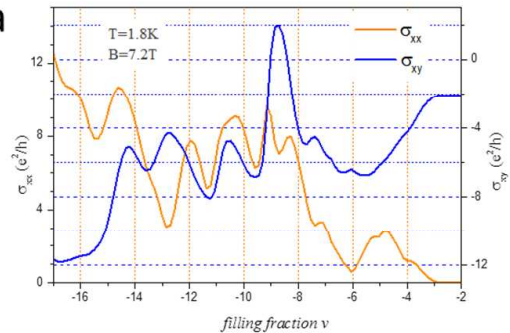

b

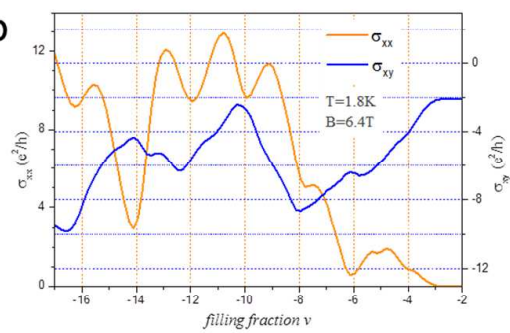

C

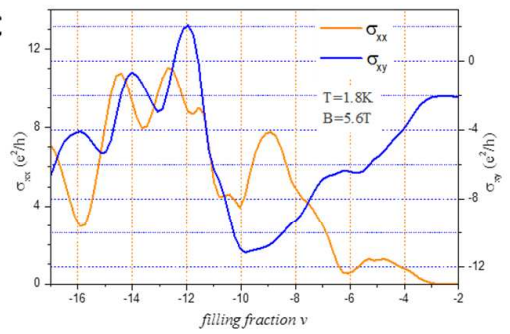

d

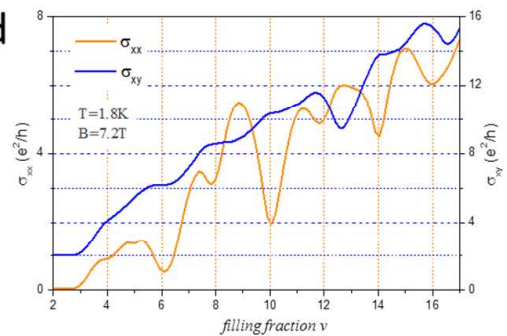

e

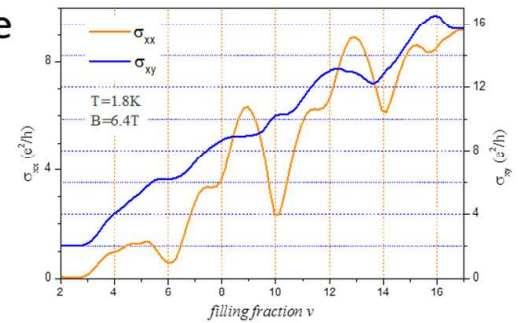

f

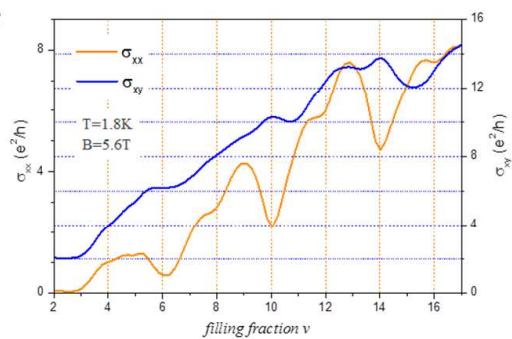

Figure S4. Conductance around magnetic flux of $\Phi=\Phi_{1 / 3}$. a-c are longitudinal conductance (yellow) as well as hall conductance (blue) at hole side with a magnetic field of 7.2T, 6.4T, and 5.6T respectively. d-f are corresponding conductance at electron side.

\section{Influence of quantum capacitance}

The geometry capacitance of our device $\mathrm{C}_{\mathrm{G}}$ is $\sim 8.2 \mathrm{nF} / \mathrm{cm}^{2}$, while the quantum capacitance per unit area $C_{Q}$ is defined as $C_{Q}=e^{2} d n / d \mu$, where $d n / d \mu$ is density of state of graphene. In the case of zero 
magnetic field, $\mathrm{dn} / \mathrm{d} \mu=8 \pi|\mathrm{E}| / \mathrm{h}^{2} \mathrm{v}_{\mathrm{F}}^{2}$, and it yields $\mathrm{C}_{\mathrm{Q}}=23 *|\mathrm{E}| \mu \mathrm{F} / \mathrm{cm}^{2}$ with $\mathrm{E}$ in the unit of $\mathrm{eV}$, which implies that $\mathrm{C}_{\mathrm{Q}}$ is small at DP and sDP. Electron-hole puddles would give a residue carrier density, for example in our device $\delta \mathrm{n}=\sim 2 * 10^{10} / \mathrm{cm}^{2}$ (corresponding to energy $\delta \mathrm{E}=\sim 15 \mathrm{meV}$ ), and it gives $\mathrm{C}_{\mathrm{Q}}=345 \mathrm{nF} / \mathrm{cm}^{2}$, which is significantly much higher that $\mathrm{C}_{\mathrm{G}}$. Thus total capacitance is mainly defined by geometry capacitance. In the case of magnetic field, the DOS is quantized according to Landau levels. Any way we can try to estimate the $\mathrm{C}_{\mathrm{Q}}$ in the vicinity of sDP at hole side using the data in Fig. $2 \mathrm{~d}$ in ref $\mathrm{f}^{8}$, which give a measured DOS of $\sim 0.1 \mathrm{eV}^{-1} \mathrm{~nm}^{-2}$ at $3 \mathrm{~T}$. Then we can roughly estimate $\mathrm{C}_{\mathrm{Q}}$ is around $1600 \mathrm{nF} / \mathrm{cm}^{2}$, which is much larger than $\mathrm{C}_{\mathrm{G}}$. To sum up, the contribution of quantum capacitance to the total capacitance is trivial, thus it can't be accounted as main reason for the deviation of constant capacitor model.

\section{Weak localization effects}

Weak localization effect is quite universal in our epitaxial graphene/hBN samples, which we mentioned before in previous paper ${ }^{9}$. For the one studied in this paper, the WL effect is evident in Figure S5, in which resistances decrease a little bit as magnetic field (before the onset of LLs) is increased, regardless of carrier doping level.

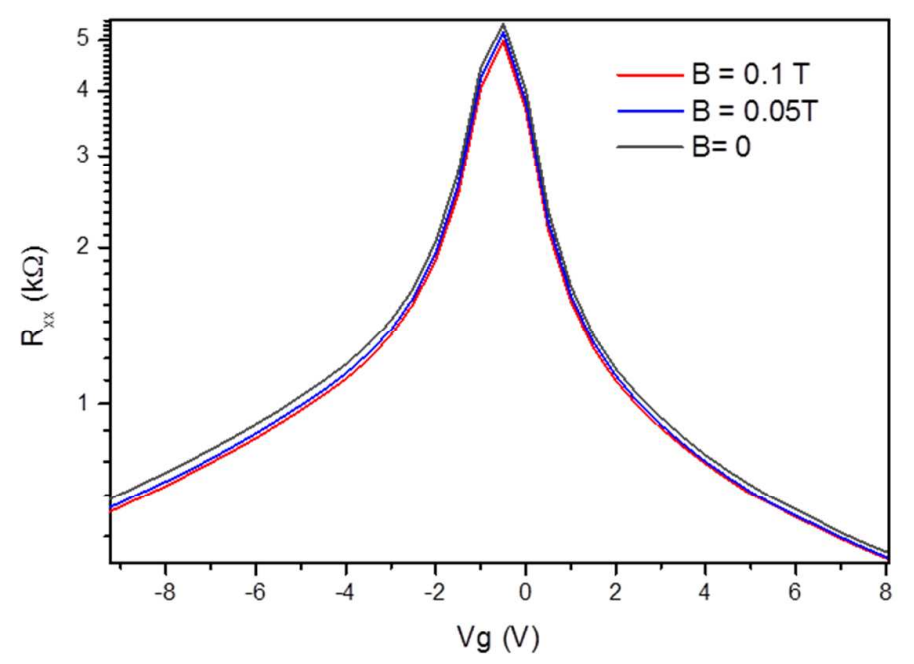

Figure S5. Transport curves at a magnetic field of $\mathrm{B}=0$ (black), $0.05 \mathrm{~T}$ (blue), $0.1 \mathrm{~T}$ (red), at a temperature of $1.8 \mathrm{~K}$.

\section{Fermi velocity measurements}

The Fermi velocity $\left(v_{F}\right)$ is estimated from cyclotron mass $(m)$ at a given magnetic field $(B)$, which in turn measured by analyzing the temperature dependence of Shubnikov-de Haas oscillations 
(SdHOs) amplitudes ${ }^{1}$. The Fermi velocity is given by $v_{F}=\hbar \sqrt{\pi n} / m$, with $n$ the carrier density defined above. The cyclotron mass $m$ is extracted from the fitting formula, $A \propto T / \sinh \left(2 \pi^{2} k_{B} m T / \hbar e B\right)$, where $A$ is SdHOs amplitude at a given temperature $(T)$ and magnetic field $(B), k_{B}$ is Boltzmann constant, and $\hbar$ is the reduced Planck constant. Take $V_{g}=20 \mathrm{~V}$ for example, the SdHOs are displayed in Figure S6. The temperature dependence of $R_{x x}$ at each resistance minima is indicated by blue arrows in Fig. S6a, and from which the amplitudes of SdHOs are extracted. Fig. S6b-e show the normalized amplitude $A(T) / A(T=1.8 K)$ at a magnetic field of $2.4 \mathrm{~T}, 3 \mathrm{~T}, 4.25 \mathrm{~T}$, and $7 \mathrm{~T}$ respectively, and the fitted curves are depicted in red. The resulting fitted cyclotron mass ( $m=m^{*} m_{e}$, where $m_{e}$ is free electron mass) as well as Fermi velocity calculated from which are then obtained and displayed as green and blue stars respectively in Fig. S6f. Clearly we could see that the $v_{F}$ decreases as magnetic field is increased, and this is true even for other gate voltages as shown in Fig. $5 \mathrm{~b}$ in the main text.
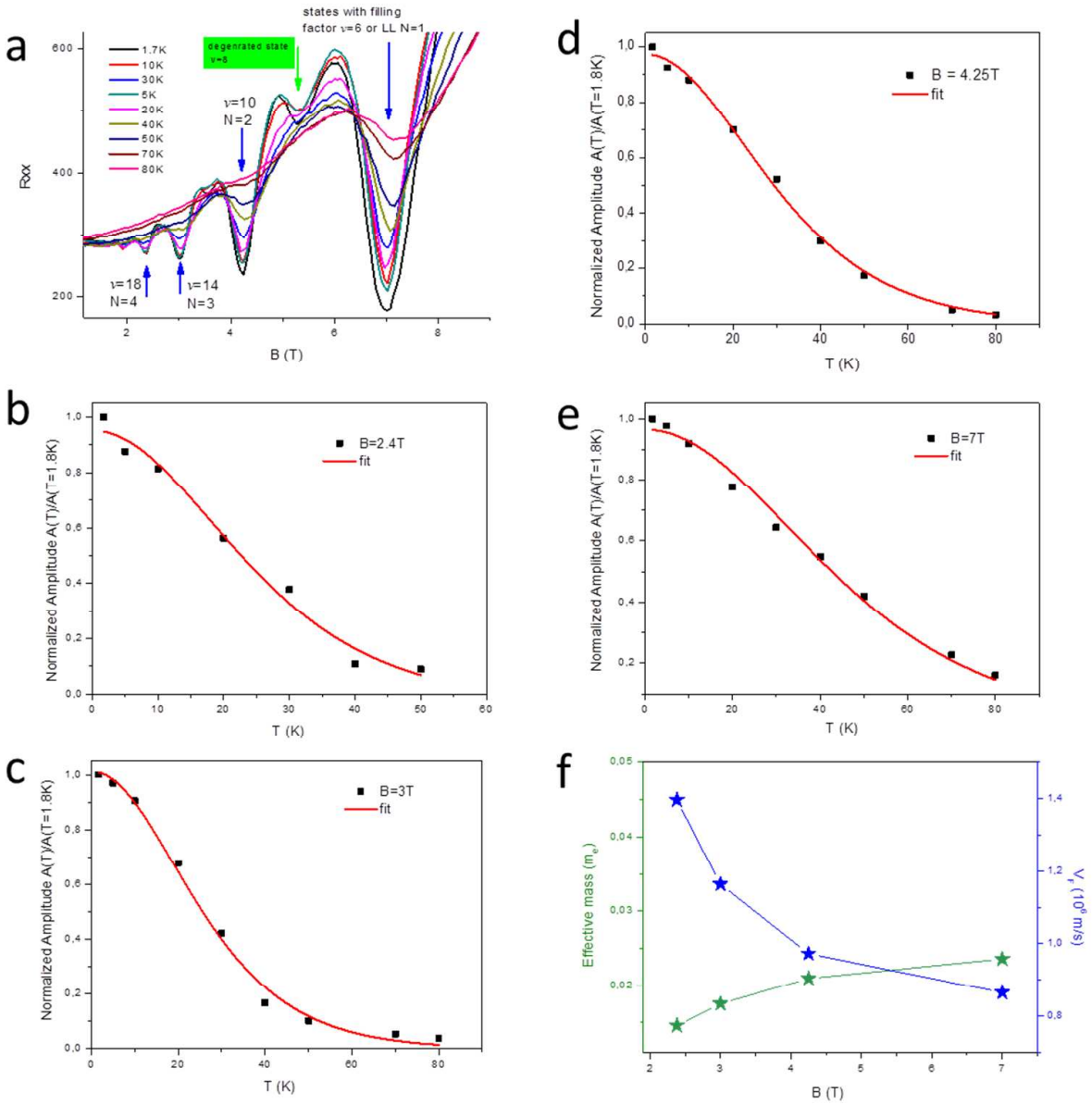
Figure S6. a, SdH Oscillations at different temperature for a gate voltage of $20 \mathrm{~V}$ away from DP, while the green arrow indicates symmetry broken states $\mathrm{v}=8$ and the blue ones the symmetry conserved states. b-e are normalized amplitudes of SdHOs at $\mathrm{B}=2.4 \mathrm{~T}, 3 \mathrm{~T}, 4.25 \mathrm{~T}$, and $7 \mathrm{~T}$ respectively. $\mathbf{f}$ is the fitted effective (cyclotron) mass as well as corresponding calculated Fermi velocity.

\section{Observation of a second generation of superlattice Dirac points}

The existence of graphene superlattice $(\lambda)$ due to the lattice mismatch between graphene and h-BN underneath produce additional Dirac points, so called superlattice Dirac points ${ }^{5-7,9-12}$, which locates at the $\mathrm{M}^{*}$ (the star denote superlattice) point of the mini-Brillouin zone (superlattice Brillouin zone, whose $\Gamma^{*}$ point is the $\mathrm{K}$ point of graphene Brillouin zone). And this is exactly what we have observed before ${ }^{9}$, and it is manifested in secondly resistance points located on both sides of main charge neutral point.

However, we only considered the effect of the first mini-Brillouin zone, and how about the case where momentum is big enough to include nearby the second mini-Brillouin zones as shown in Figure S7a (the yellow dots denote the M points of first mini-Brillouin zone, and the red ones denote $\mathrm{M}$ points of nearby second mini-Brillouin zone). We expect a second generation of superlattice Dirac points. This is a higher order effect of superlattice, and in principal it should be much weaker than that of superlattice Dirac point, so we just consider the next nearest $\mathrm{M}^{*}$ points. The nearest $\mathrm{M}^{*}$ points are indicated by yellow dots, corresponding to superlattice Dirac points (SDP), while the next-nearest $\mathrm{M}^{*}$ points to $\Gamma^{*}$ point are denoted in red, corresponding to the second generation of superlattice Dirac point (SSDP), as shown in Fig. S7a. And it is easy to see that the ratio of momentum at SSDP to that at SDP is square root of 3.
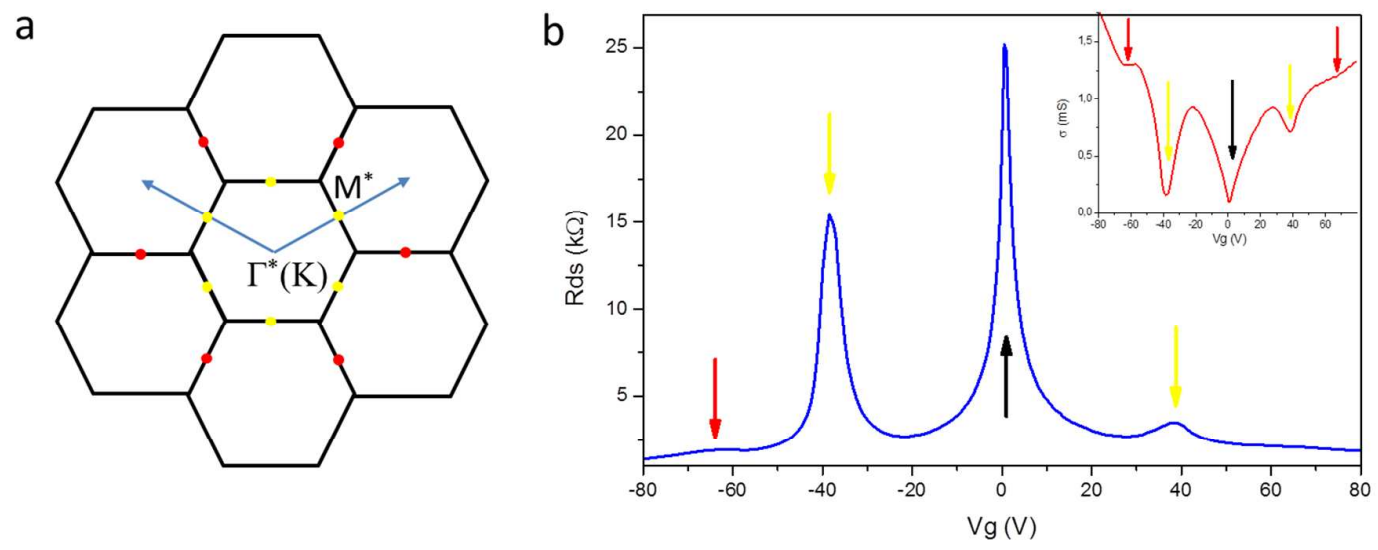

Figure S7. a, The first and nearby mini-Brillouin zone of superlattice, whose $\Gamma^{*}$ is the $\mathrm{K}$ of the Brillouin of 
graphene lattice. The yellow and red dots indicate the nearest and next-nearest $\mathrm{M}^{*}$ of mini-Brillouin zone respectively. b, Typical transport curve of a two terminal device at $\mathrm{T}=4.2 \mathrm{~K}$ without magnetic field. DP, SDP, and SSDP are indicated by black, yellow, and red arrows. The inset of $\mathbf{b}$ shows the conductivity.

We fabricate a two terminal device, with epitaxial graphene/hBN on top of a gold gate electrode (device fabricated in Laboratoire Pierre Aigrain, ENS-CNRS UMR 8551). The thickness of $\mathrm{hBN}$ is around $300 \mathrm{~nm}$, and the device geometry is $\mathrm{W} \times \mathrm{L}=3 \times 5 \mu \mathrm{m}$. The transfer curve is shown in Fig. S7b, the Dirac point is indicated by black arrow at a gate voltage of $0.5 \mathrm{~V}$, and the SDP in the first mini-Brillouin zone at hole side is indicated by yellow arrow at a gate voltage of $-38.5 \mathrm{~V}$ while the SSDP out of the first mini-Brillouin zone is indicated by red arrow at a gate voltage of $-65 \mathrm{~V}$. And it is more convenient to see the conductivity in the inset of Fig. S7b, where conductance dips at SDP and SSDP is prominent. Similar features can be found in electron side, even though they are much weaker. And note that the ratio of position at SSDP to that at SDP is $\sim 1.7$, very close to the estimated square root of 3 .

Last but not least, at high magnetic field the presence of SSDP may make the fractal Hofstadter butterfly spectra even more complicated since DP, SDP, and SSDP are not evenly separated in momentum space (or gate voltage).

\section{Reference}

1. Zhang, Y. B.; Tan, Y. W.; Stormer, H. L.; Kim, P. Nature 2005, 438, 201-204.

2. Novoselov, K. S.; Geim, A. K.; Morozov, S. V.; Jiang, D.; Katsnelson, M. I.; Grigorieva, I. V.; Dubonos, S. V.; Firsov, A. A. Nature 2005, 438, 197-200.

3. Zhang, Y.; Jiang, Z.; Small, J.; Purewal, M.; Tan, Y.-W.; Fazlollahi, M.; Chudow, J.; Jaszczak, J.; Stormer, H.; Kim, P. Phys. Rev. Lett. 2006, 96, 136806.

4. Hofstadter, D. R. Phys.Rev. B 1976, 14, 2239-2249.

5. Hunt, B.; Sanchez-Yamagishi, J. D.; Young, A. F.; Yankowitz, M.; LeRoy, B. J.; Watanabe, K.; Taniguchi, T.; Moon, P.; Koshino, M.; Jarillo-Herrero, P.; Ashoori, R. C. Science 2013, 340, 1427-1430.

6. Dean, C. R.; Wang, L.; Maher, P.; Forsythe, C.; Ghahari, F.; Gao, Y.; Katoch, J.; Ishigami, M.; Moon, P.; Koshino, M.; Taniguchi, T.; Watanabe, K.; Shepard, K. L.; Hone, J.; Kim, P. Nature 2013, 497, 598-602.

7. Ponomarenko, L. A.; Gorbachev, R. V.; Yu, G. L.; Elias, D. C.; Jalil, R.; Patel, A. A.; Mishchenko, A.; Mayorov, A. S.; Woods, C. R.; Wallbank, J. R.; Mucha-Kruczynski, M.; Piot, B. A.; Potemski, M.; Grigorieva, I. V.; Novoselov, K. S.; Guinea, F.; Fal'ko, V. I.; Geim, A. K. Nature 2013, 497, 594-597.

8. Yu, G. L.; Gorbachev, R. V.; Tu, J. S.; Kretinin, A. V.; Cao, Y.; Jalil, R.; Withers, F.; Ponomarenko, L. A.; Piot, B. A.; Potemski, M.; Elias, D. C.; Chen, X.; Watanabe, K.; Taniguchi, T.; Grigorieva, I. V.; Novoselov, K. S.; Fal/'ko, V. I.; Geim, A. K.; Mishchenko, A. Nat. Phys. 2014, 10, 525-529. 
9. Yang, W.; Chen, G.; Shi, Z.; Liu, C.-C.; Zhang, L.; Xie, G.; Cheng, M.; Wang, D.; Yang, R.; Shi, D.; Watanabe, K.; Taniguchi, T.; Yao, Y.; Zhang, Y.; Zhang, G. Nat. Mater. 2013, 12, 792-797.

10. Park, C.-H.; Yang, L.; Son, Y.-W.; Cohen, M. L.; Louie, S. G. Phys. Rev. Lett. 2008, 101, 126804.

11. Park, C. H.; Yang, L.; Son, Y. W.; Cohen, M. L.; Louie, S. G. Nat. Phys. 2008, 4, 213-217.

12. Yankowitz, M.; Xue, J. M.; Cormode, D.; Sanchez-Yamagishi, J. D.; Watanabe, K.; Taniguchi, T.; Jarillo-Herrero, P.; Jacquod, P.; LeRoy, B. J. Nat. Phys. 2012, 8, 382-386. 